\title{
Herbivoria e biomassa foliar em Peltogyne confertiflora (Mart. ex Hayne) Benth. Fabales Bromhead Fabaceae Lindl. em ambientes de Floresta e Cerrado
}

\author{
Renata D. Françoso ${ }^{1, \bowtie}$; Ana J. GoelleR ${ }^{2}$ \& Eddie Lenza ${ }^{3}$ \\ 1. Pós-Graduação em Ecologia, Instituto de Ciências Biológicas, Universidade de Brasília. Campus Universitário Darcy \\ Ribeiro, Distrito Federal, Brasil. 2. Faculdade de Ciências Sociais Aplicadas e de Tecnologias De Agua Boa. Faculdade \\ Cathedral De Ensino Superior do Araguaia, Setor Universitário, Água Boa, Mato Grosso, Brasil. 3. Universidade do Estado de \\ Mato Grosso, Departamento de Ciências Biológicas, Mato Grosso, Brasil.
}

\begin{abstract}
Resumo. Como o ambiente é um dos principais fatores responsáveis pelas características morfológicas de espécies vegetais, é esperado encontrar diferentes taxas de herbivoria em indivíduos de ambientes distintos. Neste trabalho comparamos a taxa de herbivoria foliar em Peltogyne conferti ora (Mart. ex Hayne) Benth. entre ambiente de floresta e de cerrado e avaliamos se a herbivoria é explicada por características morfológicas dos indivíduos ou pela quantidade de recurso. A taxa de herbivoria foi maior em ambiente florestal. Biomassa foliar foi selecionada como a variável mais importante para explicar a taxa de herbivoria em floresta e em cerrado. Aparentemente existe um efeito densidade-dependente na taxa de herbivoria, reforçando a necessidade da quantificação da biomassa em estudos semelhantes.
\end{abstract}

[Palavras-chave: fitofisionomias, predação foliar, interação planta-animal]

\begin{abstract}
Herbivory and leaf mass in Peltogyne confertiflora (Mart. Ex Hayne) Benth. Fabales Bromhead Fabaceae Lindl. at Forest and Cerrado: As the environment is the most important factor responsible for the morphological characteristics of plants, is expected to find differences in herbivory rates at different habitats. In this study we compared the leaf herbivory rate in Peltogyne conferti ora (Mart. ex Hayne) Benth. in forest and open vegetation (cerrado) in the Cerrado biome, and evaluated if the herbivory could be explained by individual morphological characters or by amount of resource. The herbivory rates were bigest in forest. Leaf biomass was selected as most important variable for explain herbivory rate in forest and cerrado. Apparently there is a density-dependent effect on the rate of herbivory, highlighting the need for quantification of biomass in similar studies.
\end{abstract}

[Key-words: phytophysiognomies, leaf predation, animal-plant interactions]

\section{INTRODUÇÃO}

As taxas de herbivoria variam entre grupos taxonômicos (Carmona etal. 2011), maturidade das plantas (Coley 1983; Varanda et al. 2008) e ambientes (Neves et a., 2010). Dessas interações, a variação na taxa de herbivoria entre ambientes é a mais complexa, tendo em vista a importância do ambiente como determinante da plasticidade fenotípica (Schlichting 1986).

A plasticidade fenotípica se reflete em diferenças na arquitetura, na fisiologia das plantas e na morfologia da folha (Nur \& Hasson 1984). Indivíduos submetidos a condições diversas de luminosidade, nutrientes, umidade podem apresentar diferenças na espessura, área e composição química das folhas (Dimarco et al. 2004; Peace \& Grubb 1982; Pedroso \& Varela 1995; Gurevitch et al. 2009; Gonçalves-Alvim et al. 2006; Santos \&

Editora Asociada: Adriana Salvo

$\triangle$ enatafrancoso@yahoo.com.br

Blatt 1998), resultando em diferentes níveis de consumo das folhas por herbívoros.

Por outro lado, maiores taxas de herbivoria podem estar relacionadas à disponibilidade de recurso, ou seja, o consumo foliar será maior onde houver maior densidade de indivíduos palatáveis (Root 1973). Assim, a oferta do recurso pode determinar a abundância (Lara et al. 2008) e composição da comunidade de herbívoros (Rudgers \& Whitney 2006).

Os objetivos do presente trabalho foram: i) verificar se há diferença na taxa de herbivoria foliar em Peltogyne confertiflora (Mart. ex Hayne) Benth. entre dois ambientes, e ii) avaliar se a herbivoria é explicada por características morfológicas dos indivíduos ou pela disponibilidade de recurso da espécie consumida. Para tanto, utilizamos a biomassa foliar de $P$. confertiflora como o parâmetro de quantidade de recurso.

Recibido: 16 de abril de 2013, Fin de arbitraje: 9 de julio, Nueva versión: 6 de septiembre Aceptado: 9 de septiembre. 


\section{Material e Métodos}

O estudo foi desenvolvido na Fazenda Remanso (14 $\left.47^{\circ} 50^{\prime \prime} \mathrm{S} 52^{\circ} 38^{\prime} 30^{\prime \prime} \mathrm{W}\right)$, à margem esquerda do Rio Noidori, antes da confluência com o Rio das Mortes, município de Nova Xavantina - MT. Na região há predomínio de formações savânicas do Bioma Cerrado, com trechos de formações florestais associadas aos cursos d'água. A amostragem abrange duas fitofisionomias do Cerrado, sendo uma mata ciliar (floresta) e um cerrado típico (cerrado) (sensu Ribeiro \& Walter 2008). O clima da região é do tipo Aw (tropical de savana) de Köppen, com precipitação média anual de 1600 $\mathrm{mm}$, temperaturas médias em torno de 24 a $25^{\circ} \mathrm{C}$.

\section{Nível individual}

Foram coletadas cinco folhas de 16 indivíduos de $P$. conferti ora em floresta e cinco folhas de 15 indivíduos de cerrado, totalizando 80 folhas em floresta e 75 folhas em cerrado. De cada árvore de $P$. conferti ora foram tomadas as seguintes medidas morfométricas: DAP (diâmetro a $1.30 \mathrm{~m}$ do solo) para as árvores em floresta, DAS (diâmetro a 30 $\mathrm{cm}$ do solo) para as árvores em cerrado e $\mathrm{H}$ (altura total). A luminosidade sob a copa foi estimada com o uso de um densiômetro esférico florestal com formato côncavo.

Para a estimativa da área foliar e herbivoria, folhas previamente prensadas foram fotografadas sobre um fundo contrastante, junto a uma escala de área conhecida (Figura 1A). No aplicativo ENVI 3.2 (SULSOFT $^{\circledR}$ ) as imagens foram submetidas a um classificador automático (IsoData), com no mínimo duas e no máximo três classes, gerando a classificação mais simples possível (Figura 1B). Essa classificação foi convertida para shapefile. No aplicativo ArcGis $\left(\mathrm{ESRI}^{\circledR}\right)$, usando a extensão Patch Analyst (Rempel et al. 2012), foi calculada a área de cada classe em número de pixels. Usando a área da escala como referência, a área das folhas foi obtida por proporcionalidade. Um processo de edição do arquivo foi realizado para complementar as margens das folhas onde havia lacunas, produzindo a área foliar total (Figura 1C). Após essa edição, as áreas foram calculadas novamente, e o percentual de herbivoria de cada folha foi calculado considerando a diferença entre as duas medidas. Para cada indivíduo foi calculada a média da área foliar e do percentual de herbivoria.

Para normalização dos dados, as variáveis morfométricas (altura, área foliar total e biomassa foliar) foram transformadas para logaritmo na base 10. As transformações das taxas de herbivoria e de luminosidade foram feitas pelo arco-seno da raiz da proporção. Foi feito um teste " $t$ " para comparar as taxa de herbivoria de P. conferti ora entre a Floresta e o cerrado. Para verificar se a herbivoria é explicada pelas características morfológicas, foram realizadas seleções de modelos por critério de Akaike para os indivíduos de floresta, para os indivíduos de cerrado e para todos os indivíduos em conjunto. Foi usado o software SAM (Rangel et al. 2010).

\section{Nível de comunidade}

Foram amostradas 18 parcelas em floresta e 25 no cerrado. Cada parcela tinha formato circular com raio de $315 \mathrm{~m}^{2}$, totalizando 0.57 e 0.79 há, respectivamente. Os indivíduos arbóreos de todas as espécie da comunidade foram contados e medidos (H, DAS em cerrado e DAP em floresta) para estimar a densidade arbórea e a biomassa de folhas por hectare por fitofisionomia e para o cálculo de riqueza e diversidade.

\section{Estimativa da biomassa}

Para o cálculo da biomassa em cerrado foi usada a equação $\mathrm{BV}=0.03047^{*} \mathrm{Db}^{*} 2.27159^{*} \mathrm{H} \mathrm{t}^{*} 0.89$ 48 (Rezende \& Vale 2006), onde BV( $\mathrm{t})=$ biomassa verde; $\mathrm{Db}(\mathrm{cm})=$ diâmetro do fuste a $0.30 \mathrm{~m}$ do solo e $\mathrm{Ht}(\mathrm{m})=$ altura total. Cerca de $1.5 \%$ da biomassa aérea do Cerrado corresponde à biomassa de folhas (Haridasan 2000). Para estimar a biomassa de $P$. conferti ora em floresta foi usada a equação $\mathrm{BA}=0.077+0.492 * \mathrm{DAP}^{2 *} \mathrm{Ht}$ (Higuchi et al. 1998), onde $\mathrm{BA}(\mathrm{t})=$ biomassa aérea; $\mathrm{DAP}(\mathrm{cm})=$ diâmetro a $1.20 \mathrm{~m}$ do nível do solo e $\mathrm{Ht}(\mathrm{m})=$ altura. A biomassa de folhas corresponde à $2 \%$ da biomassa total de florestas (Higuchi et al. 1998). A biomassa
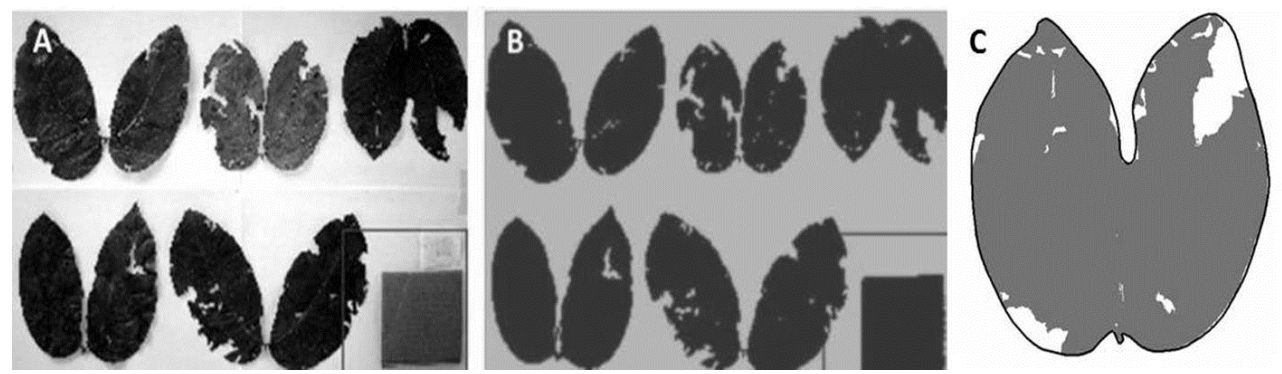

Figura 1. (A) Folhas de Peltogyne confertiflora e uma escala com área conhecida $\left(100 \mathrm{~cm}^{2}\right)$ sobre fundo contrastante. (B) Classificação automática, discriminando as folhas e a escala. (C) Área foliar existente em cinza e o complemento da borda para a estimativa da área original das folhas de Peltogyne confertiflora.

Figure 1. (A) Leaves of Peltogyne confertiflora and scale with known area $\left(100 \mathrm{~cm}^{2}\right)$ on a contrasting background. (B) Automatic classification, evidencing the leaves and scale. (C) leaf area, and drawing of edge for original area estimative of Peltogyne confertiflora. 
foi estimada tanto para a análise ao nível individual (apenas dos indivíduos de P. conferti ora amostrados) quanto para o nível de comunidade, onde foi calculada a biomassa total por hectare de floresta e cerrado.

\section{Resultados E Discussão}

\section{Herbivoria dos indivíduos}

A taxa de herbivoria tanto para indivíduos de floresta quanto para indivíduos das duas fitofisionomias simultaneamente foi explicada apenas pela biomassa foliar (Tabela 1), sendo essa a única variável do melhor modelo selecionado pelo critério de Akaike para as duas fisionomias. No cerrado o melhor modelo explicativo para a herbivoria foi biomassa foliar e área média das folhas (Tabela 1). Os demais modelos apresentados (com Delta $\mathrm{AIC}<2$ ) são igualmente prováveis, e todos eles são combinações da biomassa foliar com outras variáveis usadas.

A maior herbivoria em ambientes florestais tem sido observada em diversos estudos (Neves et al. 2010; Silva et al. 2009; Varanda \& Pais 2006), e como consequência o nível de sombreamento tem sido considerado um bom parâmetro determinar taxas de herbivoria (Peace \& Grubb 1982; Dimarco et al. 2004; Varanda \& Pais 2006). Porém, essa característica pode não determinar a herbivoria em si, mas ser apenas uma consequência do ambiente, que por outras razões levem a maiores taxas de herbivoria. No presente estudo, a variável luminosidade não foi significativa em nenhuma das análises.

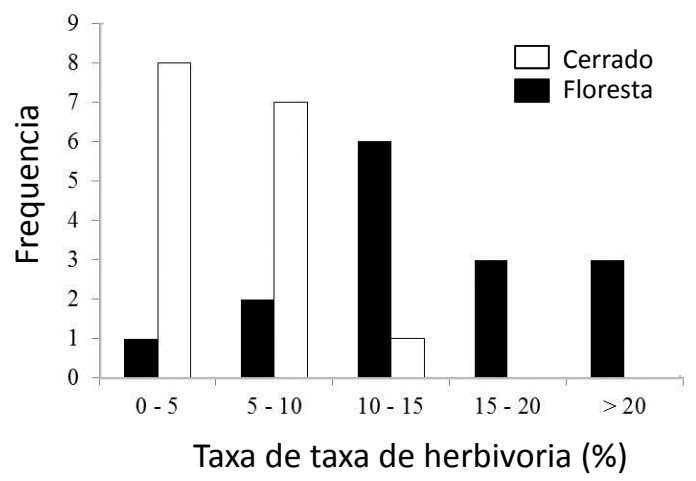

Figura 2. Frequencia da taxa de herbivoria em folhas de Peltogyne confertiflora em cerrado e floresta na fazenda Remanso, Nova Xavantina, Mato Grosso, Brasil.

Figure 2. Frequency of herbivory rate in leafs of Peltogyne confertiflora in cerrado and forest at Remanso farm, Nova Xavantina, Mato Grosso, Brasil.
Tabela 1. Modelos explicativos para a herbivoria em floresta e cerrado (junto e separadamente), na fazenda Remanso, Nova Xavantina, Mato Grosso, Brasil ordenados pelo Delta AIC de até 2. Valores em negrito representam os melhores modelos. Variáveis: $1=$ luminosidade; $2=$ altura; 3 =área foliar; $4=$ biomassa foliar. Ambientes=F- Floresta; $\mathrm{C}=$ Cerrado.

Table 1. Models explaining herbivory in forest and cerrado (separately and together), at Remanso farm, Nova Xavantina, Mato Grosso, Brasil ordered by Delta AIC until 2. See details in legend in Portuguese.

\begin{tabular}{llccccc}
\hline Ambiente Variáveis & $\mathrm{r}^{2}$ & AICc & \multicolumn{3}{c}{$\begin{array}{c}\text { Delta L(gi|x) AICw } \\
\text { AICc }\end{array}$} \\
& & \multicolumn{5}{c}{} \\
\hline $\mathrm{F}+\mathrm{C}$ & 4 & 0.936 & -120.785 & 0 & 1 & 0.372 \\
& 1,4 & 0.938 & -119.236 & 1.549 & 0.461 & 0.171 \\
& 3,4 & 0.938 & -118.938 & 1.847 & 0.397 & 0.148 \\
& 2,4 & 0.937 & -118.810 & 1.975 & 0.372 & 0.139 \\
$\mathrm{C}$ & 3,4 & 0.977 & -85.016 & 0 & 1 & 0.373 \\
& $2,3,4$ & 0.982 & -84.329 & 0.686 & 0.709 & 0.265 \\
& 4 & 0.970 & -83.891 & 1.124 & 0.57 & 0.213 \\
$\mathrm{~F}$ & 4 & 0.971 & -76.111 & 0 & 1 & 0.616 \\
\hline
\end{tabular}

A explicação corroborada pelo presente estudo é a taxa de herbivoria como consequência da disponibilidade de recurso. Diversos estudos trataram desse assunto usando área foliar (Eutrópio \& Silva 2009; Krohling et al. 2010) ou densidade de indivíduos (Bianchini \& Santos 2005), onde o efeito densidade-dependente não foi corroborado, assim como nos resultados aqui apresentados considerando essas mesmas variáveis (com exceção de área foliar no modelo do cerrado). Porém, a biomassa foliar foi eficiente para representar a quantidade de recurso, explicando de maneira significativa a taxa de herbivoria.

Variáveis morfométricas usualmente adotadas para explicação das taxas de herbivoria, como diâmetro, altura e área foliar não se mostram adequadas para explicar a herbivoria a nível individual, sendo mais recomendado o cálculo da biomassa foliar. Em nível de comunidade, a biomassa foliar também se mostrou um bom parâmetro para explicação da taxa de herbivoria. Sugerimos que outros estudos sejam realizados para verificar se a herbivoria apresenta efeito densidade-dependente também em comparações mais amplas. Para a espécie estudada não foi verificada diferença nas taxas de herbivoria como consequência da plasticidade fenotípica.

\section{Herbivoria das comunidades}

A herbivoria média na floresta foi $(15.1 \pm-6.1)$ significativamente maior do que no cerrado $(5.0 \pm 3.3)$ (Figura 2) $\left(\mathrm{t}_{28.256}=5.87, P<0.001\right)$. A 
Tabela 2. Parâmetros das comunidades vegetais em floresta e cerrado na fazenda Remanso, Nova Xavantina, Mato Grosso, Brasil.

Table2. Vegetation communities parametersin forestand cerrado at Remanso farm, Nova Xavantina, Mato Grosso, Brasil.

\begin{tabular}{lll}
\hline Variável & Floresta & Cerrado \\
\hline Índice de diversidade de & 2.194 & 3.867 \\
Shannon $\left(\mathrm{H}^{\prime}\right)$ & 65 ind & 76 ind \\
Riqueza & $49.22 \mathrm{t} / \mathrm{ha}$ & $11.35 \mathrm{t} / \mathrm{ha}$ \\
Biomassa foliar & $526 \mathrm{ind} / \mathrm{ha}$ & $776 \mathrm{ind} / \mathrm{ha}$ \\
\hline Densidade arbórea &
\end{tabular}

riqueza e abundância de herbívoros podem estar relacionadas à riqueza ou à diversidade de espécies de plantas, à disponibilidade de recursos, ou à complexidade de habitat, o que levaria a maiores taxas de herbivoria. Porém, Cirotto (2009) não observou diferença entre a composição e riqueza das comunidades de herbívoros entre floresta e cerrado. Dentre as características das comunidades estudadas, a biomassa foliar total da comunidade foi a única variável maior em floresta (49.22 ind/ha) do que no cerrado (11.35 t/ha). As demais variáveis (densidade arbórea, riqueza e diversidade) foram maiores no cerrado (Tabela 2). Dessa forma sugerimos que a disponibilidade de recurso possa explicar a maior taxa de herbivoria na floresta.

Essa diferença ainda poderia ser explicada por fatores edáficos. Plantas sobre solos mais ricos em nutrientes são menos vulneráveis e suas folhas são menos predadas (Perillo et al. 2007). Nocerrado, a heterogeneidadeambiental é explicada, dentre outras, por gradientes de solos, onde os solos sob áreas florestais são mais ricos em nutrientes do que os solos sob o cerrado (Faraco \& Cavassan 2004; Moreno et al. 2008; Haridasan 2000). Pórem, Rossato (2008) não encontrou diferenças nutricionais entre folhas de espécies congenéricas de mata e cerrado, mostrando que não há variação obrigatória na absorção de nutrientes entre essas fitofisionomias.

Agradecimentos: Agradecemos aos professores Dra Beatriz Schwantes Marimon, Dr. Ben Hur Marimon Junior, Dr. Guarino Rinaldi Colli e Dr. Reuber Albuquerque Brandão pela cooperação nos estudos realizados durante o curso de Ecologia de Campo realizado em parceria entre a Universidade de Brasília e Universidade Estadual de Mato Grosso, com financiamento do projeto PROCAD UnB/UNEMAT ( $N^{\circ}$ 109/2007).

\section{REFERÊNCIAS}

BianchinI, E \& FAM Dos SANTOS. 2005. Herbivoria Foliar em Chrysophyllum gonocarpum (Sapotaceae) no Parque Estadual Mata Dos Godoy, Londrina, Estado do Paraná, Brasil. Acta Scientiarum. Biological Sciences, 27:285-290.
Carmona, D; MJ Lajeunesse \& MTJ Johnson. 2011. Plant traits that predict resistance to herbivores. Functional Ecology, 25:358-367.

Cirotto, PA. 2009. Comparação da Fauna de Lagartas Folívoras (Lepidoptera) em Roupala montana Aubl. em Cerrado e borda de Mata de Galeria. Pp. 51.

Coley, PD. 1983. Herbivory and Defensive Characteristics of Tree Species in a Lowland Tropical Forest. Ecological Monographs, 53:209-234.

Dimarco, R; G Russo \& AG FarJI-Brener. 2004. Patrones de herbivoría en seis especies leñosas del bosque templado de América del Sur: evidencia preliminar a favor de la hipótesis del balance carbono - nutrientes. Ecologia Austral, 14: 39-43.

Eutrópio, F.J. \& A.G. Silva, 2009. Análise comparada de perda por herbivoria de área em folhas jovens e adultas de Croton sp. (Euphorbiaceae) na REBIO de Duas Bocas, Sudeste do Brasil. Natureza Online, 7:27-30.

Gonçalves-Alvim, SJ; G Korndorf \& GW Fernandes. 2006. Sclerophylly in Qualea parvi ora (Vochysiaceae): influence of herbivory, mineral nutrients, and water status. Plant Ecology, 187:153-162.

Gurevitch, J; SM Scheiner \& GA Fox. 2009. Ecologia Vegetal 2a ed., São Paulo, Artmed.

HARIDASAN, M. 2000. Nutrição mineral de plantas nativas do cerrado. Revista Brasileira de Fisiologia, 12:54-64.

Higuchi, N; J Santos; R Ribeiro; L Minette \& Y Biot. 1998. Biomassa da parte aérea da vegetação da floresta tropical úmida de terra-firme da Amazônia brasileira. Acta Amazonica, 28:153-166.

Krohling, CA; FJ Eutrópio \& AG Silva. 2010. Interações inseto-planta em Solanum hexandrum Vell. (Solanaceae): polinização e herbivoria na Reserva Biológica de Duas Bocas, Cariacica, Espirito Santo. Natureza Online, 8: 78-85.

LARA, DP; LA OliveIRA; IFP AZEVEdo; MF XAVIER; ET AL, 2008. Relationships between host plant architecture and gall abundance and survival. Revista Brasileira de Entomologia, 52:78-81.

Neves, FS; LS Araúo; MM Espírito-Santo; M Fagundes; GW FERNANDES; ET AL. 2010. Canopy Herbivory and Insect Herbivore Diversity in a Dry Forest - Savanna Transition in Brazil. Biotropica, 42:112-118.

Nur, N \& O Hasson. 1984. Phenotypic plasticity and the handicap principle. Journal of Theoretical Biology, 110: 275-297.

Peace, WJH \& PJ GrubB. 1982. Interaction of light and mineral nutrient supply in the growth of Impatiens parviflora. New Phytologist, 90:127-150.

Pedroso, SG \& VP Varela. 1995. Efeito do sombreamento no crescimento de mudas de sumauma. Revista Brasileira de Sementes, 17:47-51.

RANGel, TF; JAF Diniz-FILHo \& LM Bini. 2010. SAM: a comprehensive application for Spatial Analysis in Macroecology. Ecography, 33:46-50.

Rempel, RS; D Kaukinen \& AP Carr. 2012. Patch Analyst and Patch Grid. Ontario Ministry of Natural Resources. Centre for Northern Forest Ecosystem Research, Ontario.

Rezende, AV \& AT Vale. 2006. Comparação de modelos matemáticos para estimativa do volume, biomassa e estoque de carbono da vegetação lenhosa de um cerrado sensu stricto em Brasília, DF. Scientia Forestalis, 71:65-76. 
Root, R. 1973. Organization of a plant-arthropod association in simple and diverse habitats: the fauna of collards (Brassica oleracea). Ecological Monographs, 43:95-124.

RudGers, JA \& KD WhitNEY. 2006. Interactions between insect herbivores and a plant architectural dimorphism. Journal of Ecology, 94:1249-1260.

SAntos, MD Dos \& CTT BlatT. 1998. Teor de flavonóides e fenóis totais em folhas de Pyrostegia venusta Miers. de mata e de cerrado. Revista Brasileira de Botânica, 21:135140. SCHLichting, CD. 1986. The evolution of phenotypic plasticity in plants. Ann. Rev. Ecol. Syst., 17:667-693.

Silva, JO; FM Jesus; M Fagundes \& GW Fernandes. 2009.
Esclerofilia, taninos e insetos herbívoros associados a Copaifera lagsdorffii Desf. (Fabaceae: Caesalpinioideae) em área de transição Cerrado-Caatinga no Brasil. Ecologia Austral, 19:197-206.

VARANDA, EM; AA Costa \& JR BAROSElA. 2008. Leaf development in Xylopia aromatica (Lam) Mart. (Annonaceae): implications for palatability to Stenoma scitiorella Walker 1864 (Lepidoptera: Elachistidae). Brazilian Journal of Biology, 68:831-6.

VARANDA, EM \& MP PAIS. 2006. Insect folivory in Didymopanax vinosum (Apiaceae) in a vegetation mosaic of Brazilian cerrado. Brazilian Journal of Biology, 66:671-80. 